\title{
Nonlinear Electrodynamics Effects on the Cosmic Microwave Background: Circular Polarization
}

\author{
Herman J. Mosquera Cuesta1,2,3,4 and Gaetano Lambiase ${ }^{5,6}$ \\ ${ }^{1}$ Departamento de Física, Centro de Ciências Exatas e Tecnológicas (CCET), \\ Universidade Estadual Vale do Acaraú, Sobral, Ceará, \\ 2Instituto de Cosmologia, Relatividade e Astrofísica (ICRA-BR), \\ Centro Brasileiro de Pesquisas Físicas, Urca Rio de Janeiro, RJ, \\ 3 International Center for Relativistic Astrophysics Network (ICRANet), Pescara, \\ International Institute for Theoretical Physics and High Mathematics \\ Einstein-Galilei, PRATO, \\ 5Dipartimento di Fisica "E. R. Caianiello", \\ Universitá di Salerno, Fisciano (Sa), \\ ${ }^{6}$ INFN, Sezione di Napoli, \\ 1,2 Brazil \\ $3,4,5,6$ Italy
}

\section{Introduction}

Historically, the modifications to standard electrodynamics were introduced for preventing the appearance of infinite physical quantities in theoretical analysis involving electromagnetic interactions. For instance, Born-Infeld [1] proposed a model in which the infinite self energy of point particles (typical of linear electrodynamics) are removed by introducing an upper limit on the electric field strength and by considering the electron an electrically charged particle of finite radius. Along this line, other Lagrangians for nonlinear electrodynamics were proposed by Plebanski, who also showed that Born-Infeld model satisfy physically acceptable requirements [20], due to its feature of being inspired on the special relativity principles. Applications and consequences of nonlinear electrodynamics have been extensively studied in literature, ranging from cosmological and astrophysical models [22] to nonlinear optics, high power laser technology and plasma physics [25].

In this paper we investigate the polarization of $\mathrm{CMB}$ photons if electrodynamics is inherently nonlinear. We compute the polarization angle of CMB photons propagating in an expanding Universe, by considering in particular cosmological models with planar symmetry. It is shown that the polarization does depend on the parameter characterizing the nonlinearity of electrodynamics, which is thus constrained by making use of the recent data from WMAP and BOOMERanG (for other models see [26]). It is worth to point out that the effect we are investigating, i.e. the rotation of the polarization angle as radiation propagates in a planar geometry, is strictly related to Skrotskii effect [27]. The latter is analogous to Faraday effect 
obtained for radiation propagating in a magnetic field. The effect on the CMB radiation as predicted by NLED is polarization circular in nature. This is a unique and very distinctive feature which can be falsified with the upcoming results to be released by the PLANCK satellite collaboration.

\section{Some Lagrangian formulations of nonlinear electrodynamics}

To start with, it is worth to recall that according to quantum electrodynamics (QED: see $[7,8]$ for a complete review on NLED and QED) a vacuum has nonlinear properties (Heisenberg \& Euler 1936; Schwinger 1951) which affect the photon propagation. A noticeable advance in the realization of this theoretical prediction has been provided by [Burke, Field, Horton-Smith , etal., 1997), who demonstrated experimentally that the inelastic scattering of laser photons by gamma-rays in a background magnetic ield is definitely a nonlinear phenomenon. The propagation of photons in NLED has been examined by several authors [Bialynicka-Birula \& Bialynicki-Birula, 1970; Garcia \& Plebanski, 1989; Dittrich \& Gies, 1998; De Lorenci, Klippert, Novello, etal., 2000; Denisov, Denisova \& Svertilov, 2001a, 2001b, Denisov \& Svertilov, 2003]. In the geometric optics approximation, it was shown by [Novello, De Lorenci, Salim \& etal., 2000; Novello \& Salim, 2001], that when the photon propagation is identified with the propagation of discontinuities of the EM field in a nonlinear regime, a remarkable feature appears: The discontinuities propagate along null geodesics of an effective geometry which depends on the EM field on the background. This means that the NLED interaction can be geometrized. An immediate consequence of this NLED property is the prediction of the phenomenon dubbed as photon acceleration, which is nothing else than a shift in the frequency of any photon traveling over background electromagnetic fields. The consequences of this formalism are examined next.

\subsection{Heisenberg-Euler approach}

The Heisenberg-Euler Lagrangian for nonlinear electrodynamics (up to order 2 in the truncated infinite series of terms involving $F$ ) has the form [16]

$$
L_{\mathrm{H}-\mathrm{E}}=-\frac{1}{4} F+\bar{\alpha} F^{2}+\bar{\beta} G^{2},
$$

where $F=F_{\mu \nu} F^{\mu v}$, with $F_{\mu \nu}=\partial_{\mu} A_{\nu}-\partial_{\nu} A_{\mu}$, and $G=\frac{1}{2} \eta_{\alpha \beta \gamma \delta} F^{\alpha \beta} F^{\gamma \delta}=-4 \vec{E} \cdot \vec{B}$, with greek index running $(0,1,2,3)$, while $\bar{\alpha}$ and $\bar{\beta}$ are arbitrary constants.

When this Lagrangian is used to describe the photon dynamics the equations for the EM field in vacuum coincide in their form with the equations for a continuum medium in which the electric permittivity and magnetic permeability tensors $\epsilon_{\alpha \beta}$ and $\mu_{\alpha \beta}$ are functions of the electric and magnetic fields determined by some observer represented by its 4-vector velocity $V^{\mu}$ [Denisov, Denisova \& Svertilov, 2001a, 2001b; Denisov \& Svertilov, 2003; Mosquera Cuesta \& Salim, 2004a, 2004b]. The attentive reader must notice that this first order approximation is valid only for $B$-fields smaller than $B_{q}=\frac{m^{2} c^{3}}{e \bar{h}}=4.41 \times 10^{13} \mathrm{G}$ (Schwinger's critical $B$-field [1]). In curved spacetime, these equations are written as

$$
\begin{gathered}
D_{\| \alpha}^{\alpha}=0, \quad B_{\| \alpha}^{\alpha}=0, \\
D_{\| \beta}^{\alpha} \frac{V^{\beta}}{c}+\eta^{\alpha \beta \rho \sigma} V_{\rho} H_{\sigma \| \beta}=0,
\end{gathered}
$$




$$
B_{\| \beta}^{\alpha} \frac{V^{\beta}}{c}-\eta^{\alpha \beta \rho \sigma} V_{\rho} E_{\sigma \| \beta}=0 .
$$

Here, the vertical bars subscript " $\|$ " stands for covariant derivative and $\eta^{\alpha \beta \rho \sigma}$ is the antisymmetric Levi-Civita tensor.

The 4-vectors representing the electric and magnetic fields are defined as usual in terms of the electric and magnetic fields tensor $F_{\mu \nu}$ and polarization tensor $P_{\mu \nu}$

$$
\begin{aligned}
E_{\mu} & =F_{\mu \nu} \frac{V^{v}}{c}, & B_{\mu} & =F_{\mu \nu}^{*} \frac{V^{v}}{c}, \\
D_{\mu} & =P_{\mu \nu} \frac{V^{v}}{c}, & H_{\mu} & =P_{\mu \nu}^{*} \frac{V^{v}}{c},
\end{aligned}
$$

where the dual tensor $X_{\mu \nu}^{*}$ is defined as $X_{\mu \nu}^{*}=\frac{1}{2} \eta_{\mu v \alpha \beta} X^{\alpha \beta}$, for any antisymmetric second-order tensor $X_{\alpha \beta}$.

The meaning of the vectors $D^{\mu}$ and $H^{\mu}$ comes from the Lagrangian of the EM field, and in the vacuum case they are given by

$$
H_{\mu}=\mu_{\mu \nu} B^{v}, \quad D_{\mu}=\epsilon_{\mu \nu} E^{v},
$$

where the permeability and tensors are given as

$$
\begin{aligned}
& \mu_{\mu \nu}=\left[1+\frac{2 \alpha}{45 \pi B_{q}^{2}}\left(B^{2}-E^{2}\right)\right] h_{\mu \nu}-\frac{7 \alpha}{45 \pi B_{q}^{2}} E_{\mu} E_{v}, \\
& \epsilon_{\mu \nu}=\left[1+\frac{2 \alpha}{45 \pi B_{q}^{2}}\left(B^{2}-E^{2}\right)\right] h_{\mu \nu}+\frac{7 \alpha}{45 \pi B_{q}^{2}} B_{\mu} B_{v} .
\end{aligned}
$$

In these expressions $\alpha$ is the EM coupling constant $\left(\alpha=\frac{e^{2}}{\hbar c}=\frac{1}{137}\right)$. The tensor $h_{\mu \nu}$ is the metric induced in the reference frame perpendicular to the observers determined by the vector field $V^{\mu}$.

Meanwhile, as we are assuming that $E^{\alpha}=0$, then one gets

$$
\epsilon_{\beta}^{\alpha}=\epsilon h_{\beta}^{\alpha}+\frac{7 \alpha}{45 \pi B_{q}^{2}} B^{\alpha} B_{\beta}
$$

and $\mu_{\alpha \beta}=\mu h_{\alpha \beta}$. The scalars $\epsilon$ and $\mu$ can be read directly from Eqs. $(8,9)$ as

$$
\epsilon \equiv \mu=1+\frac{2 \alpha}{45 \pi B_{q}^{2}} B^{2} .
$$

Applying condition (8) and the method in ([14]) to the field equations when $E^{\alpha}=0$, we obtain the constraints $e^{\mu} \epsilon_{\mu v} k^{v}=0$ and $b^{\mu} k_{\mu}=0$ and the following equations for the discontinuity fields $e_{\alpha}$ and $b_{\alpha}$ :

$$
\begin{gathered}
\epsilon^{\lambda \gamma} e_{\gamma} k_{\alpha} \frac{V^{\alpha}}{c}+\eta^{\lambda \mu \rho v} \frac{V_{\rho}}{c}\left(\mu b_{v} k_{\mu}-\mu^{\prime} \lambda_{\alpha} B_{v} k_{\mu}\right)=0, \\
b^{\lambda} k_{\alpha} \frac{V^{\alpha}}{c}-\eta^{\lambda \mu \rho v} \frac{V_{\rho}}{c}\left(e_{v} k_{\mu}\right)=0 .
\end{gathered}
$$


Isolating the discontinuity field from (12), substituting in equation (13), and expressing the products of the completely anti-symmetric tensors $\eta_{\nu \zeta \gamma \beta} \eta^{\lambda \alpha \rho \mu}$ in terms of delta functions, we obtain

$$
\begin{aligned}
& b^{\lambda}\left(k_{\alpha} k^{\alpha}\right)^{2}+\left(\frac{\mu^{\prime}}{\mu} l_{\beta} b^{\beta} k_{\alpha} B^{\alpha}+\frac{\beta B_{\beta} b^{\beta} B_{\alpha} k^{\alpha}}{\mu-\beta B^{2}}\right) k^{\lambda}+ \\
& \left(\frac{\mu^{\prime}}{\mu l_{\alpha} b^{\alpha}}\left(k_{\beta} V^{\beta}\right)^{2}\left(k_{\alpha} k^{\alpha}\right)^{2}-\frac{\beta B_{\alpha} b^{\alpha}\left(k_{\beta} k^{\beta}\right)^{2}}{\mu-\beta B^{2}}\right) B^{\lambda}-\left(\frac{\mu^{\prime}}{\mu} l_{\mu} b^{\mu} k_{\alpha} B^{\alpha} k_{\beta} V^{\beta}\right) V^{\lambda}=0 .
\end{aligned}
$$

This expression is already squared in $k_{\mu}$ but still has an unknown $b_{\alpha}$ term. To get rid of it, one multiplies by $B_{\lambda}$, to take advantage of the EM wave polarization dependence. By noting that if $B^{\alpha} b_{\alpha}=0$ one obtains the dispersion relation by separating out the $k^{\mu} k^{v}$ term, what remains is the (-) effective metric. Similarly, if $B_{\alpha} b^{\alpha} \neq 0$, one simply divides by $B_{\gamma} b^{\gamma}$ so that by factoring out $k^{\mu} k^{v}$, what results is the (+) effective metric. For the case $B_{\alpha} b^{\alpha}=0$, one obtains the standard dispersion relation

$$
g^{\alpha \beta} k_{\alpha} k_{\beta}=0 .
$$

whereas for the case $B_{\alpha} b^{\alpha} \neq 0$, the result is

$$
\left[\left(1+\frac{\mu^{\prime} B}{\mu}+\frac{\tilde{\beta} B^{2}}{\mu-\tilde{\beta} B^{2}}\right) g^{\alpha \beta}-\frac{\mu^{\prime} B}{\mu} \frac{V^{\alpha} V^{\beta}}{c^{2}}+\left(\frac{\mu^{\prime} B}{\mu}+\frac{\tilde{\beta} B^{2}}{\mu-\tilde{\beta} B^{2}}\right) l^{\alpha} l^{\beta}\right] k_{\alpha} k_{\beta}=0,
$$

where $\left({ }^{\prime}\right)$ stands for $\frac{d}{d B}$, and we have defined

$$
\tilde{\beta}=\frac{7 \alpha}{45 \pi B_{q}^{2}}, \quad \text { and } \quad l^{\mu} \equiv \frac{B^{\mu}}{\left|B^{\gamma} B_{\gamma}\right|^{1 / 2}}
$$

as the unit 4-vector along the $B$-field direction.

From the above expressions we can read the effective metric $g_{+}^{\alpha \beta}$ and $g_{-}^{\alpha \beta}$, where the labels "+" and "-" refers to extraordinary and ordinary polarized rays, respectively. Then, we need the covariant form of the metric tensor, which is obtained from the expression defining the inverse metric $g_{\mu v} g^{v \alpha}=\delta_{\mu}^{\alpha}$. So that one gets from one side

$$
g_{\mu \nu}^{-}=g_{\mu \nu}
$$

and from the other

$$
\begin{array}{r}
g_{\mu \nu}^{+}=\left(1+\frac{\mu^{\prime} B}{\mu}+\frac{\beta B^{2}}{\mu-\beta B^{2}}\right)^{-1} g_{\mu v} \\
+\left[\frac{\mu^{\prime} B}{\mu\left(1+\frac{\mu^{\prime} B}{\mu}+\frac{\beta B^{2}}{\mu-\beta B^{2}}\right)\left(1+\frac{\beta B^{2}}{\mu-\beta B^{2}}\right)}\right] \frac{V_{\mu} V_{v}}{c^{2}}+\left(\frac{\frac{\mu^{\prime} B}{\mu}+\frac{\beta B^{2}}{\mu-\beta B^{2}}}{1+\frac{\mu^{\prime} B}{\mu}+\frac{\beta B^{2}}{\mu-\beta B^{2}}}\right) l_{\mu} l_{v} .
\end{array}
$$

The function $\frac{\mu^{\prime} B}{\mu}$ can be expressed in terms of the magnetic permeability of the vacuum, and is given as 


$$
\frac{\mu^{\prime} B}{\mu}=2\left(1-\frac{1}{\mu}\right)
$$

Thus equation (19) indicates that the photon propagates on an effective metric.

\subsection{Born-Infeld theory}

The propagation of light can also be viewed within the framework of the Born-Infeld Lagrangian. Such theory is inspired in the special theory of relativity, and indeed it incorporates the principle of relativity in its construction, since the fact that nothing can travel faster than light in a vacuum is used as a guide to establishing the existence of an upper limit for the strength of electric fields around an isolated charge, an electron for instance. Such charge is then forced to have a characteristic size [5]. The Lagrangian then reads

$$
L=-\frac{b^{2}}{2}\left[\left(1+\frac{F}{b^{2}}\right)^{1 / 2}-1\right] .
$$

As in this particular case, the Lagrangian is a functional of the invariant $F$, i.e., $L=L(F)$, but not of the invariant $G \equiv B_{\mu} E^{\mu}$, the study of the NLED effects turns out to be simpler (here again we suppose $E=0)$. In the equation above, $b=\frac{e}{R_{0}^{2}}=\frac{e}{\frac{e^{4}}{m_{0}^{2} c^{8}}}=\frac{m_{0}^{2} c^{8}}{e^{3}}=9.8 \times 10^{15}$ e.s.u.

In order to derive the effective metric that can be deduced from the B-I Lagrangian, one has therefore to work out, as suggested in the Appendix, the derivatives of the Lagrangian with respect to the invariant $F$. The first and second derivatives then reads

$$
L_{\mathrm{F}}=\frac{-1}{4\left(1+\frac{F}{b^{2}}\right)^{1 / 2}} \quad \text { and } \quad L_{\mathrm{FF}}=\frac{1}{8 b^{2}\left(1+\frac{F}{b^{2}}\right)^{3 / 2}} .
$$

The $L(F)$ B-I Lagrangian produces an effective contravariant metric given as

$$
g_{\text {eff }}^{\mu \nu}=\frac{-1}{4\left(1+\frac{F}{b^{2}}\right)^{1 / 2}} g^{\mu \nu}+\frac{B^{2}}{2 b^{2}\left(1+\frac{F}{b^{2}}\right)^{3 / 2}}\left[h^{\mu \nu}+l^{\mu} l^{\nu}\right] .
$$

Both the tensor $h_{\mu v}$ and the vector $l^{\mu}$ in this equation were defined earlier (see Eqs.(9) and (16) above).

Because the geodesic equation of the discontinuity (that defines the effective metric, see the Appendix) is conformal invariant, one can multiply this last equation by the conformal factor $-4\left(1+\frac{F}{b^{2}}\right)^{3 / 2}$ to obtain

$$
g_{\text {eff }}^{\mu v}=\left(1+\frac{F}{b^{2}}\right) g^{\mu v}-\frac{2 B^{2}}{b^{2}}\left[h^{\mu v}+l^{\mu} l^{\nu}\right]
$$

Then, by noting that

$$
F=F_{\mu \nu} F^{\mu v}=-2\left(E^{2}-B^{2}\right)
$$


and recalling our assumption $E=0$, then one obtains $F=2 B^{2}$. Therefore, the effective metric reads

$$
g_{\text {eff }}^{\mu v}=\left(1+\frac{2 B^{2}}{b^{2}}\right) g^{\mu v}-\frac{2 B^{2}}{b^{2}}\left[h^{\mu v}+l^{\mu} l^{\nu}\right],
$$

or equivalently

$$
g_{\text {eff }}^{\mu \nu}=g^{\mu \nu}+\frac{2 B^{2}}{b^{2}} V^{\mu} V^{v}-\frac{2 B^{2}}{b^{2}} l^{\mu} l^{\nu} .
$$

As one can check, this effective metric is a functional of the background metric $g^{\mu v}$, the 4-vector velocity field of the inertial observers $V^{v}$, and the spatial configuration (orientation $\left.l^{\mu}\right)$ and strength of the $B$-field.

Thus the covariant form of the background metric can be obtained by computing the inverse of the effective metric $g_{\text {eff }}^{\mu v}$ just derived. With the definition of the inverse metric $g_{\text {eff }}^{\mu v} g_{v \alpha}^{\text {eff }}=\delta^{\mu}{ }_{\alpha}$, the covariant form of the effective metric then reads

$$
g_{\mu v}^{\text {eff }}=g_{\mu v}-\frac{2 B^{2} / b^{2}}{\left(2 B^{2} / b^{2}+1\right)} V_{\mu} V_{v}+\frac{2 B^{2} / b^{2}}{\left(2 B^{2} / b^{2}+1\right)} l_{\mu} l_{v},
$$

which is the result that we were looking for. The terms additional to the background metric $g_{\mu \nu}$ characterize any effective metric.

\subsection{Pagels-Tomboulis Abelian theory}

In 1978, the Pagels-Tomboulis nonlinear Lagrangian for electrodynamics appeared as an effective model of an Abelian theory introduced to describe a perturbative gluodynamics model. It was intended to investigate the non trivial aspects of quantum-chromodynamics (QCD) like the asymptotic freedom and quark confinement [28]. In fact, Pagels and Tomboulis argued that:

"since in asymptotically free Yang-Mills theories the quantum ground state is not controlled by perturbation theory, there is no a priori reason to believe that individual orbits corresponding to minima of the classical action dominate the Euclidean functional integral. "

In view of this drawback, of the at the time understanding of ground states in quantum theory, they decided to examine and classify the vacua of the quantum gauge theory. To this goal, they introduced an effective action in which the gauge field coupling constant $g$ is replaced by the effective coupling $\bar{g}(t) \cdot T=\ln \left[\frac{F_{\mu \nu}^{a} F^{a} \mu v}{\mu^{4}}\right]$. The vacua of this model correspond to paramagnetism and perfect paramagnetism, for which the gauge field is $F_{\mu \nu}^{a}=0$, and ferromagnetism, for which $F_{\mu v}^{a} F^{a} \mu v=\lambda^{2}$, which implies the occurrence of spontaneous magnetization of the vacuum. ${ }^{1}$ They also found no evidence for instanton solutions to the quantum effective action. They solved the equations for a point classical source of color spin, which indicates that in the limit of spontaneous magnetization the infrared energy of the field becomes linearly divergent. This leads to bag formation, and to an electric Meissner effect confining the bag contents.

This effective model for the low energy $(3+1)$ QCD reduces, in the Abelian sector, to a nonlinear theory of electrodynamics whose density Lagrangian $L(X, Y)$ is a functional of the

\footnotetext{
${ }^{1}$ This is the imprint that such theory describes nonlinear electrodynamics.
} 
invariants $X=F_{\mu v} F^{\mu v}$ and their dual $Y=\left(F_{\mu v} F^{\mu v}\right)^{\star}$, having their equations of motion given by

$$
\nabla_{\mu}\left(-L_{X} F^{\mu v}-L_{Y}{ }^{*} F^{\mu v}\right)=0,
$$

where $L_{X}=\partial L / \partial X$ and $L_{Y}=\partial L / \partial Y$. This equation is supplemented by the Faraday equation, i. e., the electromagnetic field tensor cyclic identity (which remains unchanged)

$$
\nabla_{\mu} F_{\nu \lambda}+\nabla_{\nu} F_{\lambda \mu}+\nabla_{\lambda} F_{\mu v}=0
$$

In the case of a simple dependence on $X$, the equations of motion turn out to be [24] (here we put $C=0$ and $4 \gamma=-\left(\Lambda^{8}\right)^{(\delta-1) / 2}$ in the original Lagrangian given in [28])

$$
L_{\delta}=-\frac{1}{4}\left(\frac{X^{2}}{\Lambda^{8}}\right)^{(\delta-1) / 2} X,
$$

where $\delta$ is an dimensionless parameter and $[\Lambda]=($ an energy scale $)$. The value $\delta=1$ yields the standard Maxwell electrodynamics.

The energy-momentum tensor for this Lagrangian $L(X)$ can be computed by following the standard recipe, which then gives

$$
T_{\mu \nu}=\frac{1}{4 \pi}\left(L_{X} g^{a b} F_{\mu a} F_{b v}+g_{\mu v} L\right)
$$

while its trace turns out to be

$$
T=-\frac{1-\delta}{\pi}\left(\frac{X^{2}}{\Lambda^{8}}\right)^{(\delta-1) / 2} X
$$

It can be shown [24] that the positivity of the $T_{0}^{0} \equiv \rho$ component implies that $\delta \geq 1 / 2$.

The Lagrangian (31) has been studied by [24] for explaining the amplification of the primordial magnetic field in the Universe, being the analysis focused on three different regimes: 1) $B^{2} \gg$ $E^{2}$, 2) $B^{2} \simeq \mathcal{O}\left(E^{2}\right)$, 3) $E^{2} \ll B^{2}$. It has also been used by [23] to discuss both the origin of the baryon asymmetry in the universe and the origin of primordial magnetic fields. More recently it has also been discussed in the review on "Primordial magneto-genesis" by [17].

Because the equation of motion (29) above, exhibits similar mathematical aspect as eq. (35) (reproduced in the Section), it appears clear that the Pagels and Tomboulis Lagrangian (31) leads also to an effective metric identical to that one given in equation (40), below.

\subsection{Novello-Pérez Bergliaffa-Salim NLED}

More recently, [21] Novello, Pérez Bergliaffa, Salim revisited the several general properties of nonlinear electrodynamics by assuming that the action for the electromagnetic field is that of Maxwell with an extra term, namely ${ }^{2}$

$$
S=\int \sqrt{-g}\left(-\frac{F}{4}+\frac{\gamma}{F}\right) d^{4} x
$$

\footnotetext{
${ }^{2}$ Notice that this Lagrangian is gauge invariant, and that hence charge conservation is guaranteed in this theory.
} 
where $F \equiv F_{\mu \nu} F^{\mu \nu}$.

Physical motivations for bringing in this theory have been provided in [21]. Besides of those arguments, an equally unavoidable motivation comes from the introduction in the 1920's of both the Heisenberg-Euler and Born-Infeld nonlinear electrodynamics discussed above, which are valid in the regime of extremely high magnetic field strengths, i.e. near the Schwinger's limit. Both theories have been extensively investigated in the literature (see for instance [22] and the long list of references therein). Since in nature non only such very strong magnetic fields exist, then it appears to be promising to investigate also those super weak field frontiers. From the conceptual point of view, this phenomenological action has the advantage that it involves only the electromagnetic field, and does not invoke entities that have not been observed (like scalar fields) and/or speculative ideas (like higher-dimensions and brane worlds).

At first, one notices that for high values of the field $F$, the dynamics resembles Maxwell's one except for small corrections associate to the parameter $\gamma$, while at low strengths of $F$ it is the $1 / F$ term that dominates. (Clearly, this term should dramatically affect, for instance, the photon- $\vec{B}$ field interaction in intergalactic space, which is relevant to understand the solution to the Pioneer anomaly using NLED.). The consistency of this theory with observations, including the recovery of the well-stablished Coulomb law, was shown in [21] using the cosmic microwave radiation bound, and also after discussing the anomaly in the dynamics of Pioneer 10 spacecraft [22]. Both analysis provide small enough values for the coupling constant $\gamma$.

\subsubsection{Photon dynamics in NPS NLED: Effective geometry}

Next we investigate the effects of nonlinearities in the evolution of EM waves in the vacuum permeated by background $\vec{B}$-fields. An EM wave is described onwards as the surface of discontinuity of the EM field. Extremizing the Lagrangian $L(F)$, with $F\left(A_{\mu}\right)$, with respect to the potentials $A_{\mu}$ yields the following field equation [20]

$$
\nabla_{v}\left(L_{F} F^{\mu v}\right)=0,
$$

where $\nabla_{v}$ defines the covariant derivative. Besides this, we have the EM field cyclic identity

$$
\nabla_{\nu} F^{* \mu \nu}=0 \quad \Leftrightarrow \quad F_{\mu v \mid \alpha}+F_{\alpha \mu \mid v}+F_{\nu \alpha \mid \mu}=0 .
$$

Taking the discontinuities of the field Eq.(35) one gets (all the definitions introduced here are given in [14] $)^{3}$

$$
L_{F} f_{\lambda}^{\mu} k^{\lambda}+2 L_{F F} F^{\alpha \beta} f_{\alpha \beta} F^{\mu \lambda} k_{\lambda}=0,
$$

which together with the discontinuity of the Bianchi identity yields

$$
f_{\alpha \beta} k_{\gamma}+f_{\gamma \alpha} k_{\beta}+f_{\beta \gamma} k_{\alpha}=0 .
$$

\footnotetext{
${ }^{3}$ Following Hadamard's method [15], the surface of discontinuity of the EM field is denoted by $\Sigma$. The field is continuous when crossing $\Sigma$, while its first derivative presents a finite discontinuity. These properties are specified as follows: $\left[F_{\mu v}\right]_{\Sigma}=0, \quad\left[F_{\mu v \mid \lambda}\right]_{\Sigma}=f_{\mu v} k_{\lambda}$, where the symbol $\left[F_{\mu v}\right]_{\Sigma}=$ $\lim _{\delta \rightarrow 0^{+}}\left(\left.J\right|_{\Sigma+\delta}-\left.J\right|_{\Sigma-\delta}\right)$ represents the discontinuity of the arbitrary function $J$ through the surface $\Sigma$. The tensor $f_{\mu \nu}$ is called the discontinuity of the field, $k_{\lambda}=\partial_{\lambda} \Sigma$ is the propagation vector, and the symbols "|" and "||" stand for partial and covariant derivatives.
} 
A scalar relation can be obtained if we contract this equation with $k^{\gamma} F^{\alpha \beta}$, which yields

$$
\left(F^{\alpha \beta} f_{\alpha \beta} g^{\mu v}+2 F^{\mu \lambda} f_{\lambda}^{v}\right) k_{\mu} k_{v}=0 .
$$

It is straightforward to see that here we find two distinct solutions: a) when $F^{\alpha \beta} f_{\alpha \beta}=0$, case in which such mode propagates along standard null geodesics, and b) when $F^{\alpha \beta} f_{\alpha \beta}=\chi$. In the case a) it is important to notice that in the absence of charge currents, this discontinuity describe the propagation of the wave front as determined by the field equation (35), above. Thence, following [19] the quantity $f^{\alpha \beta}$ can be decomposed in terms of the propagation vector $k_{\alpha}$ and a space-like vector $a_{\beta}$ (orthogonal to $k_{\alpha}$ ) that describes the wave polarization. Thus, only the light-ray having polarization and direction of propagation such that $F^{\alpha \beta} k_{\alpha} a_{\beta}=0$ will follow geodesics in $g_{\mu \nu}$. Any other light-ray will propagate on the effective metric (40). Meanwhile, in this last case, we obtain from equations (37) and (39) the propagation equation for the field discontinuities being given by [22]

$$
\underbrace{\left(g^{\mu \nu}-4 \frac{L_{F F}}{L_{F}} F^{\mu \alpha} F_{\alpha}^{v}\right)}_{\text {effective metric }} k_{\mu} k_{v}=0 .
$$

This equation proves that photons propagate following a geodesic that is not that one on the background space-time, $g^{\mu \nu}$, but rather they follow the effective metric given by Eq.(40), which depends on the background field $F^{\mu \alpha}$, i. e., on the $\vec{B}$-field.

\section{Minimally coupling gravity to nonlinear electrodynamics}

The action of (nonlinear) electrodynamics coupled minimally to gravity is

$$
S=\frac{1}{2 \kappa} \int d^{4} x \sqrt{-g} R+\frac{1}{4 \pi} \int d^{4} x \sqrt{-g} L(X, Y),
$$

where $\kappa=8 \pi G, L$ is the Lagrangian of nonlinear electrodynamics depending on the invariant $X=\frac{1}{4} F_{\mu \nu} F^{\mu v}=-2\left(\mathbf{E}^{2}-\mathbf{B}^{2}\right)$ and $Y=\frac{1}{4} F_{\mu \nu}{ }^{*} F^{\mu v}$, where $F^{\mu v} \equiv \nabla_{\mu} A_{v}-\nabla_{v} A_{\mu}$, and ${ }^{*} F^{\mu v}=\epsilon^{\mu v \rho \sigma} F_{\rho \sigma}$ is the dual bivector, and $\epsilon^{\alpha \beta \gamma \delta}=\frac{1}{2 \sqrt{-g}} \varepsilon^{\alpha \beta \gamma \delta}$, with $\varepsilon^{\alpha \beta \gamma \delta}$ the Levi-Civita tensor $\left(\varepsilon_{0123}=+1\right)$.

The equations of motion are

$$
\nabla_{\mu}\left(-L_{X} F^{\mu v}-L_{Y}{ }^{*} F^{\mu v}\right)=0,
$$

where $L_{X}=\partial L / \partial X$ and $L_{Y}=\partial L / \partial Y$,

$$
\nabla_{\mu} F_{\nu \lambda}+\nabla_{\nu} F_{\lambda \mu}+\nabla_{\lambda} F_{\mu \nu}=0
$$

After a swift grasp on this set of equations one realizes that is difficult to find solutions in closed form of these equations. Therefore to study the effects of nonlinear electrodynamics, we confine ourselves to consider the abelian Pagels-Tomboulis theory [28], proposed as an effective model of low energy QCD. The Lagrangian density of this theory involves only the invariant $X$ in the form

$$
L(X)=-\left(\frac{X^{2}}{\Lambda^{8}}\right)^{\frac{\delta-1}{2}} X=-\gamma X^{\delta},
$$


where $\gamma($ or $\Lambda$ ) and $\delta$ are free parameters that, with appropriate choice, reproduce the well known Lagrangian already studied in the literature. $\gamma$ has dimensions [energy] ${ }^{4(1-\delta)}$.

The equation of motion for the Pagels-Tomboulis theory follows from Eq. (42) with $Y=0$

$$
\nabla_{\mu} F^{\mu v}=-(\delta-1) \frac{\nabla_{\mu} X}{X} F^{\mu v} .
$$

In terms of the potential vector $A^{\mu}$, and imposing the Lorentz gauge $\nabla_{\mu} A^{\mu}=0$, Eq. (45) becomes

$$
\nabla_{\mu} \nabla^{\mu} A^{v}+R_{\mu}^{v} A^{\mu}=-(\delta-1) \frac{\nabla_{\mu} X}{X}\left(\nabla^{\mu} A^{v}-\nabla^{v} A^{\mu}\right),
$$

where the Ricci tensor $R_{\mu}^{v}$ appears because the relation $\left[\nabla^{\mu}, \nabla_{v}\right] A^{v}=-R_{\mu}^{\mu} A^{\mu}$.

We work in the geometrical optics approximation (this means that the scales of variation of the electromagnetic fields are smaller than the cosmological scales), so that the 4 -vector $A^{\mu}(x)$ can be written as [29]

$$
A^{\mu}(x)=\operatorname{Re}\left[\left(a^{\mu}(x)+\epsilon b^{\mu}(x)+\ldots\right) e^{i S(x) / \epsilon}\right]
$$

with $\epsilon \ll 1$ so that the phase $S / \epsilon$ varies faster than the amplitude. By defining the wave vector $k_{\mu}=\nabla_{\mu} S$, which defines the direction of the photon propagation, one finds that the gauge condition implies $k_{\mu} a^{\mu}=0$ and $k_{\mu} b^{\mu}=0$. It turns out to be convenient to introduce the normalized polarization vector $\varepsilon^{\mu}$ so that the vector $a^{\mu}$ can be written as

$$
a^{\mu}(x)=A(x) \varepsilon^{\mu}, \quad \varepsilon_{\mu} \varepsilon^{\mu}=1 .
$$

As a consequence of (48), one also finds $k_{\mu} \varepsilon^{\mu}=0$, i.e. the wave vector is orthogonal to the polarization vector.

To leading order in $\epsilon$, the relevant equations are

$$
(2 \delta-1) k^{2}=0 \rightarrow k_{\mu} k^{\mu}=0,
$$

for $\delta \neq \frac{1}{2}$, hence photons propagate along null geodesics, and

$$
k^{\mu} \nabla_{\mu} \varepsilon^{\sigma}=\frac{\delta-1}{\delta} k_{\mu}\left[\nabla^{\sigma} \varepsilon^{\mu}-\left(\varepsilon^{\rho} \nabla_{\rho} \varepsilon^{\mu}\right) \varepsilon^{\sigma}\right] .
$$

\section{Space-time anisotropy and magnetic energy density evolution}

In what follows we consider cosmological models with planar symmetry

$$
d s^{2}=d t^{2}-b^{2}\left(d x^{2}+d y^{2}\right)-c^{2} d z^{2},
$$

where $b(t)$ and $c(t)$ are the scale factors, which are normalized in order that $b\left(t_{0}\right)=1=c\left(t_{0}\right)$ at the present time $t_{0}$. As Eq. (51) shows, the symmetry is on the (xy)-plane.

We shall assume that photons propagate along the (positive) $x$-direction, so that $k^{\mu}=$ $\left(k^{0}, k^{1}, 0,0\right)$. Gauge invariance assures that the polarization vector of photons has only two independent components, which are orthogonal to the direction of the photons motion. 
By defining the affine parameter $\lambda$ which measures the distance along the line-element, $k^{\mu} \equiv$ $d x^{\mu} / d \lambda$, one obtains that $\varepsilon^{2}$ and $\varepsilon^{3}$ satisfy the following geodesic equation (from Eq. (50))

$$
\begin{aligned}
& \frac{1}{k^{0}} \mathcal{D} \ln \left(b \varepsilon^{2}\right)=-\frac{\delta-1}{\delta}\left(-\frac{\dot{b}}{b}+\frac{\dot{c}}{c}\right)\left(c \varepsilon^{3}\right)^{2}, \\
& \frac{1}{k^{0}} \mathcal{D} \ln \left(c \varepsilon^{3}\right)=-\frac{\delta-1}{\delta}\left(-\frac{\dot{c}}{c}+\frac{\dot{b}}{b}\right)\left(b \varepsilon^{2}\right)^{2} .
\end{aligned}
$$

where $\mathcal{D} \equiv k^{\mu} \nabla_{\mu}$. Moreover, the difference of the Hubble expansion rate $\dot{b} / b$ and $\dot{c} / c$ can be written as

$$
\frac{\dot{b}}{b}-\frac{\dot{c}}{c}=\frac{1}{2\left(1-e^{2}\right)} \frac{d e^{2}}{d t}
$$

where we have introduced the eccentricity

$$
e(t)=\sqrt{1-\left(\frac{c}{b}\right)^{2}}
$$

The polarization angle $\alpha$ is defined as $\alpha=\arctan \left[\left(c \varepsilon^{3}\right) /\left(b \varepsilon^{2}\right)\right]$. By introducing the reference time $t$, corresponding to the moment in which photons are emitted from the last scattering surface, and the instant $t_{0}$, corresponding to the present time, one finally gets

$$
\Delta \alpha=\alpha(t)-\alpha\left(t_{0}\right)=\frac{\delta-1}{4 \delta} K e^{2}(z),
$$

where $K$ is a constant. Here we have used $e\left(t_{0}\right)=0$, because of the normalization condition $b\left(t_{0}\right)=c\left(t_{0}\right)=1$, and $\log \left(1-e^{2}\right) \sim-e^{2}$.

Notice that for $\delta=1$ or $e^{2}=0$ there is no rotation of the polarization angle, as expected. Moreover, in the case in which photons propagate along the direction $z$-direction, so that $\bar{k}^{a}=\left(\omega_{0}, 0,0, k\right)$, we find that the NLED have no effects as concerns to the rotation of the polarization angle.

\subsection{Eccentricity evolution on cosmic time}

The time evolution of the eccentricity is determined from the Einstein field equations

$$
\frac{1}{1-e^{2}} \frac{d(e \dot{e})}{d t}+3 H_{b}(e \dot{e})+\frac{(e \dot{e})^{2}}{\left(1-e^{2}\right)^{2}}=2 \kappa \rho_{B},
$$

where $H_{b}=\dot{b} / b$ and

$$
\rho_{B}=\frac{B^{2}}{8 \pi}\left(\frac{B^{2}}{2 \Lambda^{4}}\right)^{\delta-1}
$$

It is extremely difficult to exactly solve this equation. We shall therefore assume that the $e^{2}$-terms can be neglected. Since $b(t) \sim t^{2 / 3}$ during the matter-dominated era, Eq. (57) implies

$$
e^{2}(z)=18 F_{\delta}(z) \Omega_{B}^{(0)},
$$

where we used $1+z=b\left(t_{0}\right) / b(t), e\left(t_{0}\right)=0$, and

$$
F_{\delta} \equiv \frac{3}{(9-8 \delta)(4 \delta-3)}-2-\frac{3(1+z)^{4 \delta-3}}{(9-8 \delta)(4 \delta-3)}+2(1+z)^{\frac{3}{2}} .
$$


$\Omega_{B}^{(0)}$ is the present energy density ratio

$$
\begin{gathered}
\Omega_{B}^{(0)}=\frac{\rho_{B}}{\rho_{c r}}=\frac{B^{2}\left(t_{0}\right)}{8 \pi \rho_{c r}}\left(\frac{B^{2}\left(t_{0}\right)}{2 \Lambda^{4}}\right)^{\delta-1} \simeq \\
10^{-11}\left(\frac{B\left(t_{0}\right)}{10^{-9} \mathrm{G}}\right)^{2}\left(\frac{B^{2}\left(t_{0}\right)}{2 \Lambda^{4}}\right)^{\delta-1},
\end{gathered}
$$

with $\rho_{c r}=3 H_{b}^{2}\left(t_{0}\right) / \kappa=8.1 h^{2} 10^{-47} \mathrm{GeV}^{4}$ ( $h=0.72$ is the little- $h$ constant), and $B\left(t_{0}\right)$ is the present magnetic field amplitude.

From Eq. (56) then follows

$$
\Delta \alpha=\frac{\delta-1}{4 \delta} K e^{2}\left(z_{\text {dec }}\right) .
$$

where $e\left(z_{\text {dec }}\right)^{2}$ the eccentricity (59) evaluated at the decoupling $z=1100$.

\subsection{Constraints on extragalactic B strengths}

To make an estimate on the parameter $\delta$, we need the order of amplitude of the present magnetic field strength $B\left(t_{0}\right)$. In this respect, observations indicate that there exist, in cluster of galaxies, magnetic fields with field strength $\left(10^{-7}-10^{-6}\right) \mathrm{G}$ on $10 \mathrm{kpc}-1 \mathrm{Mpc}$ scales, whereas in galaxies of all types and at cosmological distances, the order of magnitude of the magnetic field strength is $\sim 10^{-6} \mathrm{G}$ on (1-10) kpc scales. The present accepted estimations is (see for example [30])

$$
B\left(t_{0}\right) \lesssim 10^{-9} \mathrm{G} .
$$

Moreover, for an ellipsoidal Universe the eccentricity satisfies the relation $0 \leq e^{2}<1$. The condition $e^{2}>0$ means $F_{\delta}>0$, with $F_{\delta}$ defined in (60). The function $F_{\delta}$ given by Eq. (60) is represented in Fig. 1. Clearly the allowed region where $F_{\delta}$ is positive does depend on the redshift $z$. On the other hand, the condition $e^{2}<1$ poses constraints on the magnetic field strength. By requiring $e^{2}<10^{-1}$ (in order that our approximation to neglect $e^{2}$-terms in (57) holds), from Eqs. (59)-(61) it follows

$$
B\left(t_{0}\right) \lesssim 9 \times 10^{-8} \mathrm{G} .
$$

It must also be noted that such magnetic fields does not affect the expansion rate of the universe and the $\mathrm{CMB}$ fluctuations because the corresponding energy density is negligible with respect to the energy density of CMB.

\section{Stokes parameters, rotated CMB spectra and constraints on parameter $\Lambda$}

The propagation of photons can be described in terms of the Stokes parameters $I, Q, U$, and $V$. The parameters $Q$ and $V$ can be decomposed in gradient-like $(G)$ and a curl-like (C) components [31] ( $G$ and $C$ are also indicated in literature as $E$ and $B$ ), and characterize the orthogonal modes of the linear polarization (they depend on the axes where the linear polarization are defined, contrarily to the physical observable $I$ and $V$ which are independent on the choice of coordinate system). 

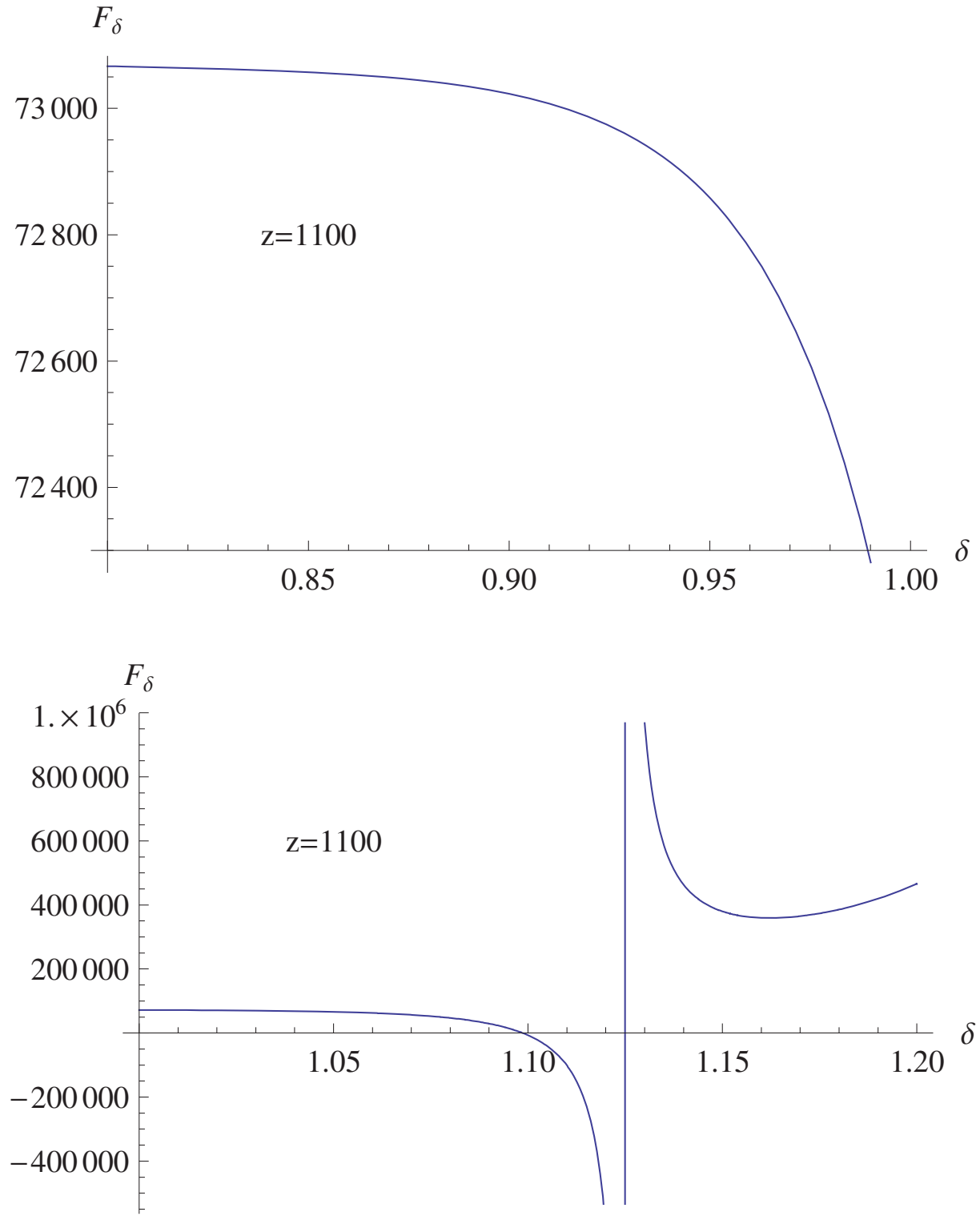

Fig. 1. In this plot is represented $F_{\delta}$ vs $\delta$ for $\delta \leq 1$ (upper plot) and $\delta \geq 1$ (lower plot). The condition that the eccentricity is positive follows for $F_{\delta}>0$. 
The polarization $G$ and $C$ and the temperature $(T)$ are crucial because they allow to completely characterize the $\mathrm{CMB}$ on the sky. If the Universe is isotropic and homogeneous and the electrodynamics is the standard one, then the TC and GC cross-correlations power spectrum vanish owing to the absence of the cosmological birefringence. In presence of the latter, on the contrary, the polarization vector of each photons turns out to be rotated by the angle $\Delta \alpha$, giving rise to $T C$ and $G C$ correlations.

Using the expression for the power spectra $C_{l}^{X Y} \sim \int d k\left[k^{2} \Delta_{X}\left(t_{0}\right) \Delta_{Y}\left(t_{0}\right)\right]$, where $X, Y=$ $T, G, C$ and $\Delta_{X}$ are the polarization perturbations whose time evolution is controlled by the Boltzman equation, one can derive the correlation for $T, G$ and $C$ in terms of $\Delta \alpha$ [32]

$$
\begin{gathered}
C_{l}^{\prime T C}=C_{l}^{T C} \sin 2 \Delta \alpha, \quad C_{l}^{\prime T G}=C_{l}^{T G} \cos 2 \Delta \alpha, \\
C_{l}^{\prime G C}=\frac{1}{2}\left(C_{l}^{G G}-C_{l}^{C C}\right) \sin 4 \Delta \alpha, \\
C_{l}^{\prime G G}=C_{l}^{G G} \cos ^{2} 2 \Delta \alpha+C_{l}^{C C} \sin ^{2} 2 \Delta \alpha, \\
C_{l}^{\prime C C}=C_{l}^{C C} \cos ^{2} 2 \Delta \alpha+C_{l}^{G G} \sin ^{2} 2 \Delta \alpha .
\end{gathered}
$$

The prime indicates the rotated quantities. Notice that the $\mathrm{CMB}$ temperature power spectrum remains unchanged under the rotation. Experimental constraints on $\Delta \alpha$ have been put from the observation of CMB polarization by WMAP and BOOMERanG

$$
\Delta \alpha=(-2.4 \pm 1.9)^{\circ}=[-0.0027 \pi,-0.0238 \pi] .
$$

The combination of Eqs. (69) and (62), and the laboratory constraints $|\delta-1| \ll 1$ allow to estimate $\Lambda$.

\subsection{Estimative of $\Lambda$}

To estimate $\Lambda$ we shall write

$$
\begin{aligned}
B & =10^{-9+b} \mathrm{G} & & b \lesssim 2, \\
F_{\delta} & =2 z^{3 / 2} & & z=1100 \gg 1 .
\end{aligned}
$$

The bound (69) can be therefore rewritten in the form

$$
\frac{10^{-3}}{\mathcal{A}} \lesssim|\delta-1| \lesssim \frac{10^{-2}}{\mathcal{A}}
$$

where

$$
\begin{gathered}
\mathcal{A} \equiv \frac{9 K}{14} F_{\delta} \Omega_{B}^{(0)} \simeq \\
\simeq K \times 10^{-6+2 b}\left[0.24 \times 10^{-56+2 b}\left(\frac{\mathrm{GeV}}{\Lambda}\right)^{4}\right]^{\delta-1} .
\end{gathered}
$$

The condition $|\delta-1| \ll 1$ requires $\mathcal{A} \gg 1$. Setting $\mathcal{A}=10^{a}$, with $a>\mathcal{O}(1)$, it follows

$$
\log \left[\frac{\Lambda}{\mathrm{GeV}}\right]=\left(-14+\frac{b}{2}\right)-\frac{a-2 b+6-\log K}{4(\delta-1)} .
$$



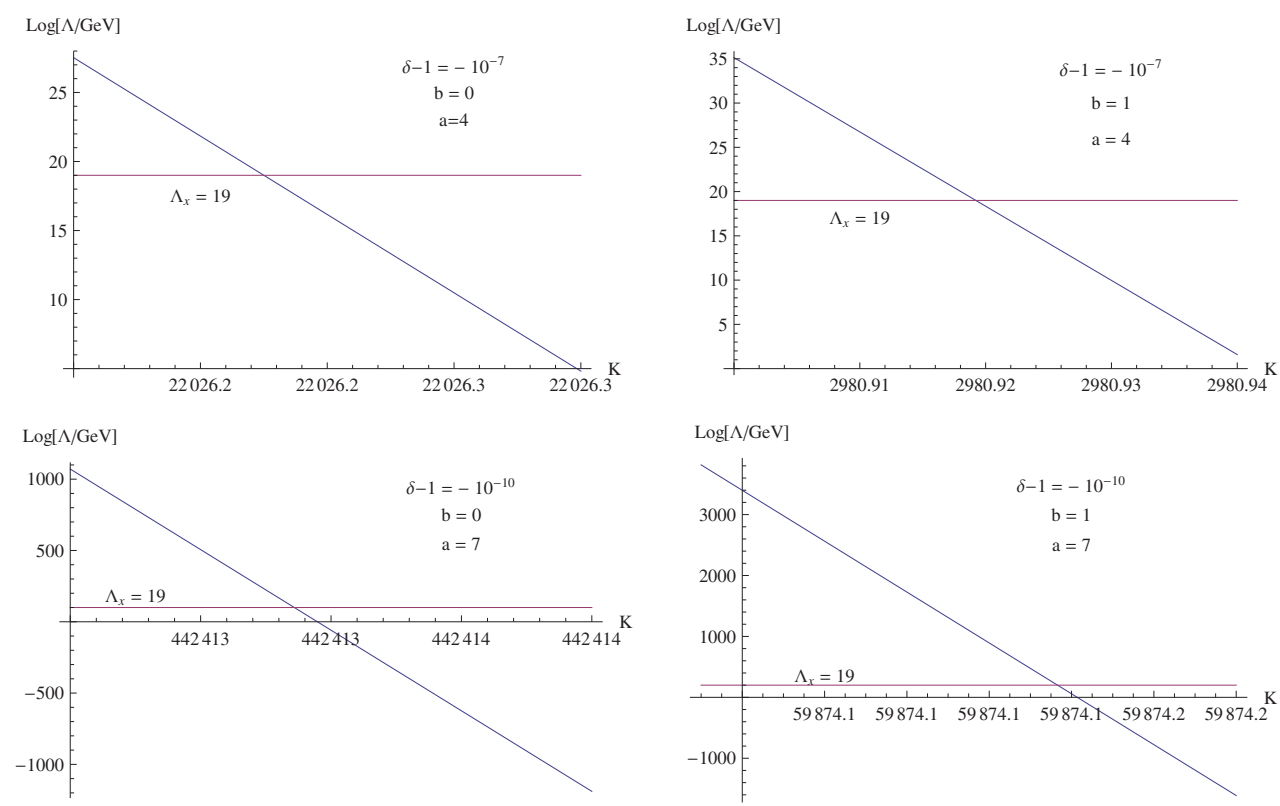

Fig. 2. $\Lambda$ vs $K$ for different values of the parameter $\delta-1, a$ and $b$. The parameter $a$ is related to the range in which $\delta-1$ varies, i.e. $-10^{-3-a} \lesssim \delta-1 \lesssim-10^{-2-a}$, while $b$ parameterizes the magnetic field strength $B=10^{-9+b} \mathrm{G}$. The red-shift is $z=1100$. Plot refers to Planck scale $\Lambda=10^{\Lambda_{x}} \mathrm{GeV}$, with $\Lambda_{x=P l}=19$. Similar plots can be also obtained for GUT $\left(\Lambda_{\mathrm{GUT}}=16\right)$ and $\mathrm{EW}\left(\Lambda_{\mathrm{EW}}=3\right)$ scales.

The constant $K$ can now be determined to fix the characteristic scale $\Lambda$. Writing $\Lambda=10^{\Lambda_{x}}$ $\mathrm{GeV}$, where $\Lambda_{x=P l}=19$, i.e. $\Lambda$ is fixed by Planck scale, Eq. (74) yields

$$
\begin{aligned}
K & =10^{a-2 b+6-\zeta}, \\
\zeta & \equiv \frac{4(\delta-1) \Lambda_{x}}{14-b / 2} \ll 1 .
\end{aligned}
$$

In Fig. 2 is plotted $\log (\Lambda / \mathrm{GeV})$ vs $K$ for fixed values of the parameters $a, b$ and $\delta-1$. Similar plots can be derived for GUT and EW scales.

\section{Discussion and future perspective}

In conclusion, in this paper we have calculated, in the framework of the nonlinear electrodynamics, the rotation of the polarization angle of photons propagating in a Universe with planar symmetry. We have found that the rotation of the polarization angle does depend on the parameter $\delta$, which characterizes the degree of nonlinearity of the electrodynamics. This parameter can be constrained by making use of recent data from WMAP and BOOMERang. Results show that the CMB polarization signature, if detected by future CMB observations, would be an important test in favor of models going beyond the standard model, including the nonlinear electrodynamics. 
Moreover, an interesting topic that deserves to be studied is the possibility to generate a circular polarization in nonlinear electrodynamics. As well know there is at the moment no mechanism to generate it at the last scattering, provided some extension of the standard electrodynamics [33]. Recently, Motie and Xue [34] have discussed the photon circular polarization in the framework of Euler-Heisenberg Lagrangian. It is therefore worthwhile to derive a circular polarization in the framework of the Pagels-Tomboulis nonlinear electrodynamics described by (44) and for a background described by nonplanar geometry. It is expected, however, that the effects are very small [35].

As closing remark, we would like to point out that the approach to analyze the CMB polarization in the context of NLED that we have presented above can also be applied to discuss the extreme-scale alignments of quasar polarization vectors [36], a cosmic phenomenon that was discovered by Hutsemekers[37] in the late 1990's, who presented paramount evidence for very large-scale coherent orientations of quasar polarization vectors (see also Hutsemekers and Lamy [38]). As far as the authors of the present paper are awared of, the issue has remained as an open cosmological connundrum, with a few workers in the field having focused their attention on to those intriguing observations [35].

\section{Appendix}

\section{A. Light propagation in NLED and birefringence}

In this Appendix we shortly discuss the modification of the light velocity (birefringence effect) for the model of nonlinear electrodynamics $L(X)$. According to [39], one finds that the effect of birefringent light propagation in a generic model for nonlinear electrodynamics is given by the optical metric

$$
\begin{aligned}
& g_{1}^{\mu v}=\mathcal{X} g^{\mu v}+(\mathcal{Y}+\sqrt{\mathcal{Y}-\mathcal{X} \mathcal{Z}}) t^{\mu v}, \\
& g_{2}^{\mu v}=\mathcal{X} g^{\mu v}+(\mathcal{Y}-\sqrt{\mathcal{Y}-\mathcal{X} \mathcal{Z}}) t^{\mu v},
\end{aligned}
$$

where the quantities $\mathcal{X}, \mathcal{Y}$, and $\mathcal{Z}$ are related to the derivatives of the Lagrangian $L(X)$ and $t^{\mu \nu}=F^{\mu \alpha} F_{\alpha}^{v}$. For our model, expressed by Eq. (44), we infer $\left(K_{1}=L_{X}, K_{2}=8 L_{X X}\right)$ [29]

$$
g_{1}^{\mu \nu}=K_{1}\left(K_{1} g^{\mu \nu}+2 K_{2} t^{\mu \nu}\right), \quad g_{2}^{\mu \nu}=K_{1}^{2} g^{\mu \nu} .
$$

which show that birefringence is present. This means that some photons propagate along the standard null rays of spacetime metric $g^{\mu \nu}$, whereas other photons propagate along rays null with respect to the optical metric $K_{1} g^{\mu \nu}+2 K_{2} t^{\mu \nu}$.

The velocities of the light wave can be derived by using the light cone equations (effective metric). The value of the mean velocity, obtained by averaging over the directions of propagation and polarization, is given by

$$
\begin{aligned}
\left\langle v^{2}\right\rangle & \simeq 1+(\delta-1) R+(\delta-1)^{2} S, \\
R & \equiv \frac{4}{3} \frac{T^{00}}{\left[4 X+2(\delta-1) t^{00}\right]}, \\
S & =\frac{4}{3} \frac{S^{2}}{\left[4 X+2(\delta-1) t^{00}\right]^{2}},
\end{aligned}
$$


where $\mathbf{S}$ is the energy flux density.

The high accuracy of optical experiments in laboratories requires tiny deviations from standard electrodynamics. This condition is satisfied provided $|\delta-1| \ll 1$.

\section{References}

[1] M. Born, Nature (London) 132, 282 (1933); Proc. R. Soc. A 143, 410 (1934). M. Born, L. Infeld, Nature (London) 132, 970 (1933); Proc. R. Soc. A 144, 425 (1934). W. Heisenberg, H. Euler, Z. Phys. 98, 714 (1936). J. Schwinger, Phys. Rev. 82, 664 (1951).

[2] Bialynicka-Birula, Z. Bialynicki-Birula, I. (1970). Phys. Rev. D 2, 2341

[3] Blasi, P. \& Olinto, A. V. (1999). Phys. Rev. D 59, 023001

[4] Burke, D. L., et al. (1997). Phys. Rev. Lett. 79, 1626

[5] Born, M. \& Infeld, L. (1934). Proc. Roy. Soc. Lond. A 144, 425

[6] De Lorenci, V. A.., Klippert, R., Novello, M. \& Salim, M., (2002). Phys. Lett. B 482, 134

[7] Delphenich, D. H. (2003). Nonlinear electrodynamics and QED. arXiv: hep-th/0309108

[8] Delphenich, D. H. (2006). Nonlinear optical analogies in quantum electrodynamics. arXiv: hep-th/0610088

[9] Denisov, V. I., Denisova, I. P. \& Svertilov, S. I. (2001a). Doklady Physics, Vol. 46, 705.

[10] Denisov, V. I., Denisova, I. P., Svertilov, S. I. (2001b). Dokl. Akad. Nauk Serv. Fiz. 380, 435

[11] Denisov, V. I., Svertilov, S. I. (2003). Astron. Astrophys. 399, L39

[12] Dittrich, W. \& Gies, H. (1998). Phys Rev.D 58, 025004

[13] Garcia, A.. \& Plebanski, J. (1989). J. Math. Phys. 30, 2689

[14] Hadamard, J. (1903) Leçons sur la propagation des ondes et les equations de l'Hydrodynamique (Hermann, Paris, 1903)

[15] Following Hadamard [14], the surface of discontinuity of the EM field is denoted by $\Sigma$. The field is continuous when crossing $\Sigma$, while its first derivative presents a finite discontinuity. These properties are specified as follows: $\left[F_{\mu \nu}\right]_{\Sigma}=0,\left[F_{\mu v \mid \lambda}\right]_{\Sigma}=f_{\mu v} k_{\lambda}$, where the symbol $\left[F_{\mu \nu}\right]_{\Sigma}=\lim _{\delta \rightarrow 0^{+}}\left(\left.J\right|_{\Sigma+\delta}-\left.J\right|_{\Sigma-\delta}\right)$ represents the discontinuity of the arbitrary function $J$ through the surface $\Sigma$. The tensor $f_{\mu \nu}$ is called the discontinuity of the field, $k_{\lambda}=\partial_{\lambda} \Sigma$ is the propagation vector, and symbols "|" and "॥" stand for partial and covariant derivatives.

[16] Heisenberg, W. \& Euler, H., (1936). Zeit. Phys. 98, 714

[17] Kandus, A.., Kerstin, K. E. \& Tsagas, C. (2010). Primordial magneto-genesis. arXiv: 1007.3891 [astro-ph.CO]

[18] Landau, L. D. \& Lifchiftz, E. (1970). Théorie des Champs. (Editions MIR, Moscou)

[19] Lichnerowicz, A., (1962). Elements of Tensor Calculus, (John Wiley and Sons, New York). See also Relativistic Hydrodynamics and Magnetohydrodynamics (W. A. Benjamin, 1967), and Magnetohydrodynamics: Waves and Shock Waves in Curved Space-Time (Kluwer, Springer, 1994)

[20] J.F. Plebanski, Lectures on nonlinear electrodynamics, monograph of the Niels Bohr Institute Nordita, Copenhagen (1968).

[21] M. Novello, S.E. Pérez Bergliaffa, J. Salim, Phys. Rev. D 69, 127301 (2004). See also V. A. De Lorenci et al., Phys. Lett. B 482: 134-140 (2000).

[22] H.J. Mosquera Cuesta, J.M. Salim, M. Novello, arXiv:0710.5188 [astro-ph]. L. Campanelli, P. Cea, G.L. Fogli, L. Tedesco, Phys. Rev. D 77, 043001 (2008); Phys. Rev. D 77, 123002 
(2008). H. J. Mosquera Cuesta and J. M. Salim, MNRAS 354, L55 (2004). H. J. Mosquera Cuesta and J. M. Salim, ApJ 608, 925 (2004). H. J. Mosquera Cuesta, J. A. de Freitas Pacheco and J. M. Salim, IJMPA 21, 43 (2006) J-P. Mbelek, H. J. Mosquera Cuesta, M. Novello and J. M. Salim Eur. Phys. Letts. 77, 19001 (2007). J. P. Mbelek, H. J. Mosquera Cuesta, MNRAS 389, 199 (2008).

[23] H.J. Mosquera Cuesta, G. Lambiase, Phys. Rev. D 80, 023013 (20009).

[24] K.E. Kunze, Phys. Rev. D 77, 023530 (2008).

[25] M. Marklund, P.K. Shukla, Rev. Mod. Phys. 78, 591 (2006). J. Lundin, G. Brodin, M. Marklund, Phys. of Plasmas 13: 102102 (2006). E. Lundstrom et al. Phys. Rev. Lett. 96 083602 (2006).

[26] J-Q Xia, H. Li, X. Zhang, arXiv:0908.1876v2 [astro-ph.CO].

[27] G.V. Strotskii, Dokl. Akad. Nauk. SSSR 114, 73 (1957) [Sov. Phys. Dokl. 2, 226 (1957)].

[28] H. Pagels, E. Tomboulis, Nucl. Phys. B 143, 485 (1978).

[29] H. Mosquesta Cuesta and G. Lambiase, JCAP 1103 (2011) 033.

[30] J.D. Barrow, R. Marteens, Ch.G. Tsagas, Phys. Rep. 449, 131 (2007).

[31] M. Kamionkowski, A. Kosowski, A. Stebbins, Phys. Rev. D 55, 7368 (1997).

[32] G. Gubitosi, L. Pagano, G. Amelino-Camelia, A. Melchiorri, A. Cooray, JCAP 0908, 021 (2009). M. Das, S. Mohanty, A.R. Prasanna, arXiv:0908.0629v1[astro-ph.CO]. P. Cabella, P. Natoli, J. Silk, Phys. Rev. D 76, 123014 (2007). F.R. Urban, A.R. Zhitnitsky, arXiv:1011.2425 [astro-ph.CO]. G.-C. Liu, S. Lee, K.-W. Ng, Phys. Rev. Lett. 97, 161303 (2006). Y.-Z. Chu, D.M. Jacobs, Y. Ng, D. Starkman, Phys. Rev. D 82, 064022 (2010). S. di Serego Alighieri, arXiv:1011.4865 [astro-ph.CO]. R. Sung, P. Coles, arXiv:1004.0957v1[astro-ph.CO].

[33] M. Giovannini, Phys. Rev. D 81, 023003 (2010); Phys. Rev. D 80, 123013 (2009). M. Giovannini and K.E. Kunze, Phys. Rev. D 78, 023010 (2008). S. Alexander, J. Ochoa, and A. Kosowsky, Phys. Rev. D 79, 063524 (2009). M. Zarei, E. Bavarsad, M. Haghighat, R. Mohammadi, I. Motie, and Z. Rezaei, Phys. Rev. D 81, 084035 (2010). F. Finelli and M. Galaverni, Phys. Rev. D 79, 063002 (2009). A. Cooray, A. Melchiorri, and J. Silk, Phys. Lett. B 554, 1 (2003).

[34] I. Motie and S.-S. Xue, arXiv:1104.3555 [hep-ph].

[35] H. Mosquesra Cuesta and G. Lambiase, in preparation (2011).

[36] D. Hutsemekers, R. Cabanac, H. Lamy, D. Sluse, Astron.Astrophys. 441, 915 (2005).

[37] D. Hutsemekers, Astron. Astrophys 332, 410 (1998).

[38] D. Hutsemekers, H. Lamy, Astron. Astrophys 358, 835 (2000).

[39] Y.N. Obukhov, G.F. Rubilar, Phys. Rev. D 66, 024042 (2002). 


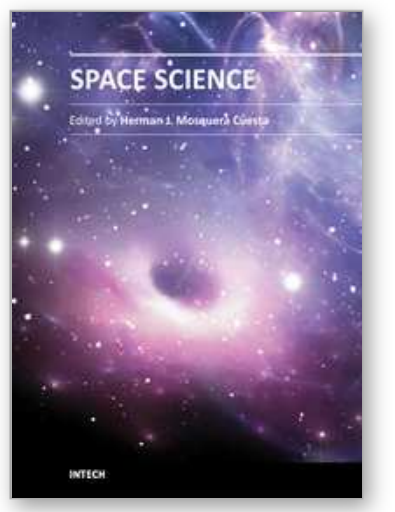

\author{
Space Science \\ Edited by Dr. Herman J. Mosquera Cuesta
}

ISBN 978-953-51-0423-0

Hard cover, 152 pages

Publisher InTech

Published online 23, March, 2012

Published in print edition March, 2012

The all-encompassing term Space Science was coined to describe all of the various fields of research in science: Physics and astronomy, aerospace engineering and spacecraft technologies, advanced computing and radio communication systems, that are concerned with the study of the Universe, and generally means either excluding the Earth or outside of the Earth's atmosphere. This special volume on Space Science was built throughout a scientifically rigorous selection process of each contributed chapter. Its structure drives the reader into a fascinating journey starting from the surface of our planet to reach a boundary where something lurks at the edge of the observable, light-emitting Universe, presenting four Sections running over a timely review on space exploration and the role being played by newcomer nations, an overview on Earth's early evolution during its long ancient ice age, a reanalysis of some aspects of satellites and planetary dynamics, to end up with intriguing discussions on recent advances in physics of cosmic microwave background radiation and cosmology.

\title{
How to reference
}

In order to correctly reference this scholarly work, feel free to copy and paste the following:

Herman J. Mosquera Cuesta and Gaetano Lambiase (2012). Nonlinear Electrodynamics Effects on the Cosmic Microwave Background: Circular Polarization, Space Science, Dr. Herman J. Mosquera Cuesta (Ed.), ISBN: 978-953-51-0423-0, InTech, Available from: http://www.intechopen.com/books/space-science/nonlinearelectrodynamics-effects-on-the-cosmic-microwave-background-radiation-polarization

\section{INTECH}

open science | open minds

\section{InTech Europe}

University Campus STeP Ri

Slavka Krautzeka 83/A

51000 Rijeka, Croatia

Phone: +385 (51) 770447

Fax: +385 (51) 686166

www.intechopen.com

\section{InTech China}

Unit 405, Office Block, Hotel Equatorial Shanghai

No.65, Yan An Road (West), Shanghai, 200040, China

中国上海市延安西路65号上海国际贵都大饭店办公楼405单元

Phone: +86-21-62489820

Fax: +86-21-62489821 
(C) 2012 The Author(s). Licensee IntechOpen. This is an open access article distributed under the terms of the Creative Commons Attribution 3.0 License, which permits unrestricted use, distribution, and reproduction in any medium, provided the original work is properly cited. 